\title{
Fiesta peninsular y holiday estadounidense: dos visiones
}

\author{
The american concept of holiday versus its spanish counterpart: two perspectives
}

\author{
Ángel Raluy Alonso \\ Universidad de Vic, Facultad de Educación, Traducción y Humanidades, \\ Departamento de Filología, Barcelona, España. Correo electrónico: raluy4@hotmail.com
}

Este artículo parte de la hipótesis de que la palabra fiesta posee un peso semántico mayor que su correspondiente holiday norteamericana y que esta desigualdad se puede corroborar en el ámbito de la antropología, la literatura, la lexicografía y la semántica lingüística. La falta de equivalencia semántica de ambos vocablos puede ocasionar dificultades en encuentros interculturales, ya que la traducción literal no consigue transmitir las diversas connotaciones semánticas de la fiesta. Convendría, pues, evitar tanto que el estudiante hispanohablante vuelque en el sustantivo estadounidense sus nociones culturales festivas, como que el estudiante norteamericano de español reduzca el término lingüístico al colorismo de una celebración lúdica o incluso taurina.

Palabras clave: fiesta, semántica intercultural, lexicografía, lingüística comparada.

This paper presents the hypothesis that the word fiesta has a greater semantic weight than its American counterpart holiday. That lack of cognitive equivalence will be analysed from an anthropologic, literary, lexicographic and semantic point of view. That different conception may interfere in intercultural communication since a literal translation cannot possibly reflect the diverse connotations of the noun fiesta. Both the Spanish student of English and the North American student of Spanish could benefit from understanding the cultural notions associated with these key words to avoid stereotypes and linguistic oversimplifications.

Key words: Holiday, intercultural semantics, lexicography, comparative linguistics.

\section{LA FIESTA EN LAS COLONIAS AMERICANAS}

Desde la perspectiva antropológica norteamericana parece predominar la definición de la fiesta como tiempo aparte ligado a ocasiones preestablecidas: "Por fiesta entiendo un día, unos días o un periodo de tiempo socialmente reconocido con el propósito de celebrar un evento del pasado como un santo, acción de gracias, navidad, el cumpleaños de George Washington, año nuevo o para señalar la transición de estaciones”" (Santino 1994: XXVIII).

"By holiday I mean a socially recognized day or period of days set aside to celebrate an important person or event of the past, such as saints' days, Thanksgiving, Christmas, or Washington's birthday, or to mark a transition of some kind, such as the beginning of the new year or the turning of the season". 
Daniel Bell (1979: 105) también define la fiesta como un momento de ruptura de la cotidianidad que puede entrar en conflicto con la ética puritana del trabajo. $\mathrm{Su}$ formulación es clara: sin dedicación exclusiva al trabajo no se fomenta la productividad, sin productividad no se genera crecimiento económico y sin éste el sistema capitalista se ve abocado al desastre. Para Bell, en la fiesta subyace una irracionalidad que hace peligrar la marcha de la sociedad y de sus instituciones más serias como la ciencia, la economía o la política.

Esta cosmovisión contemporánea tiene sus raíces en la herencia puritana que configuró la vida en las colonias americanas desde sus orígenes. Ciertamente, la violenta reacción luterana contra los excesos de la fiesta en la iglesia y en la sociedad contribuyó poderosamente a su descrédito en las colonias del nuevo mundo: se limitaron los días de asueto e incluso se llegaron a prohibir juegos y espectáculos: "Es cierto que los puritanos consideraban los festejos populares de su tiempo como demoníacos... lo que proporcionaba sosiego, alegría y diversión a los puritanos estaba a años luz del regocijo y la frivolidad de las fiestas"”2 (Stephenson 1979: 32).

Los gobernantes de Nueva Inglaterra aspiraban a crear unas reglas universales con las que regir la sociedad y se les hacía intolerable la idea del mundo de la fiesta donde las normas sociales se ponían en entredicho. De hecho, algunas escisiones del protestantismo americano (cuáqueros y congregacionistas) pretendieron borrar del calendario todas las fiestas (incluso el día de Navidad estuvo en peligro) y concentrarlas en el domingo con un sentido de férrea religiosidad.

Esta primera etapa de represión fue perdiendo fuerza hacia la primera mitad del siglo XVIII, debido tanto a una mejora de las condiciones económicas como a las masivas oleadas de inmigrantes no puritanos en su mayoría hostiles a la rigidez social reinante. Aun así, el puritanismo continuó tiñendo la fiesta de una ambivalencia moral que no consiguió borrar el capitalismo industrial del siglo XIX. Filósofos y escritores de este período no cesaban de formular críticas contra el poder de la fiesta. Así, Emerson (2004: 16) resaltó la animalidad y la pérdida de control que se reproduce en la fiesta en cuanto fenómeno social: "existe siempre esa circunstancia vindicativa al acecho incluso en la salvaje poesía con la cual el ser humano intentó resaltar la fiesta". ${ }^{3}$ La misma actitud de rechazo manifiesta Henry Adams (2002: 58). La novela, verdadero manual de costumbres, menciona los peligros de la fiesta desde una perspectiva cotidiana: "Si los muchachos tuviesen fiesta, serían presa de las trampas del Thiergarten o de cualquier otro lugar, siempre con el resultado de agotamiento, fumareda, salchichas y cerveza". ${ }^{4}$ El desarrollo capitalista, no obstante, comportará una paulatina pérdida del significado religioso de holiday, que se verá sustituido por un sentido de descanso laboral, que tampoco gozará de la aprobación de la sociedad americana ni tampoco de su literatura. Connotaciones de frivolidad y hasta podría decirse falta de responsabilidad se atisban en la obra de Edith Warton (1998: 777): "Dada la ligereza de sus obligaciones profesionales, habría sido

\footnotetext{
"It is true that the Puritans regarded the popular amusements of the day as of the devil...what gave peace, joy, and amusement to the Puritan was leagues removed from the gaiety and frivolity of holidays."

"there is always this vindictive circumstance stealing in at unawares, even into the wild poesy in which the human fancy attempted to make bold holiday.”

$4 \quad$ "If the boys had a holiday, they were apt to be taken on long tramps in the Thiergarten or elsewhere, always ending in over-fatigue, tobacco-smoke, sausages, and beer.”
} 
condenado por frivolidad por el clan Mingott si hubiera sugerido pedir fiesta en invierno". 5

Tras la primera guerra mundial, la fiesta y el tiempo libre se fueron democratizando y su presencia se fue extendiendo a capas cada vez más amplias de la sociedad estadounidense, aunque desde una perspectiva capitalista exacerbada. El consumismo pasó a ser la nueva celebración norteamericana con el beneplácito de la incipiente industria del entretenimiento: "La semana corta está a punto de llegar ya que sin ella este país no será capaz de absorber su producción y continuar siendo próspero... es la influencia del ocio en el consumo lo que hace necesario acortar los días y las semanas laborables”6 (Ibrahim 1991: 75).

Las últimas décadas del siglo XX han visto un cierto incremento en el tiempo de diversión y en las opciones de fiesta, pero sigue subyaciendo una visión crítica del ocio, como resalta Updyke (1970: 24), quien se atrevió a definirlo como “ la amenaza más siniestra al bienestar del trabajador y tema de creciente preocupación por parte de los sindicatos"?

\section{LA FIESTA EN EsPaña}

La antropología española se muestra radicalmente diferente. Martínez-Burgos (2004: 291) afirma que la fiesta es el espacio donde la comunidad cobra relieve y la considera un elemento clave en la organización de la actividad social ya que renueva los vínculos entre individuos. La dualidad trabajo/fiesta no tiene razón de ser porque la fiesta es razón del trabajo. Gil (1998: 341) reafirma la ligazón histórica entre la cultura productiva y la festiva al señalar que, ya en la Edad Media, la península gozaba de una media de 115 días de fiesta al año que sumados a los 52 domingos alcanzaban la cifra de 167 días. Esta tendencia festiva se reafirmó durante los primeros reinados de los Austria, pero su consagración como fenómeno social llegaría en la España del Barroco: ostentación pública y excesos lúdicos que tenían como objeto ocultar el marasmo que vivía el estado en el siglo XVII. Como dice Chalmeta (1995: 80) citando a Gracián: "La fiesta era una farsa con muchas tramoyas y apariencias, célebre espectáculo en medio de aquel gran teatro de todo el mundo”.

Los excesos festivos del barroco español fueron violentamente criticados por los pensadores y escritores del siglo XVIII que culpaban a la fiesta de la situación de postración en la que se encontraba el país. Bajo el lema "menos fiestas y más trabajo", se promulgó una serie de decretos de censura de la fiesta pública que culminaron en el reinado de Carlos III con la temporal prohibición de las corridas y las comedias. No obstante, ni los ataques del despotismo ilustrado ni los vientos de cambio que impuso el radicalismo revolucionario en la vecina Francia, penetraron con suficiente fuerza en la península ibérica como para desprestigiar la fiesta, que continuó siendo

"Little arduous as his professional duties were, he would have been convicted of frivolity by the whole Mingott clan if he had suggested asking for a holiday in mid-winter.”

6 "The short week is bound to come, because without it the country will not be able to absorb its production and stay prosperous... but it is the influence of leisure on consumption which makes the short day and the short week so necessary.”

"the darkest threat to the well-being of the working man and the subject of increasing concern on the part of organized labor". 
el motor de la existencia, por encima incluso de necesidades básicas: "Sí, respondió Pedro; pero hay fiesta, y se lo llevarán allá los amigos: siempre ha sido tan bailador, que dejaría la comida por un fandango" (Caballero 1998: 65). A partir de la segunda mitad del siglo XIX se inició un proceso de secularización y racionalidad que hizo mella en la fiesta, al menos formalmente. La incipiente exaltación del trabajo señaló a la ociosidad festiva como madre de todos los vicios y propuso modelos de fiesta productiva como modelo de regeneración social. En este sentido, la introducción de la economía capitalista de mercado en España significó un aumento de la jornada laboral y en consecuencia un recorte de los días festivos. Aunque esta reducción nunca fue tan drástica en la península ibérica como en las sociedades anglosajonas, es cierto que las necesidades de producción ayudaron a reducir el número de jornadas festivas del calendario. La literatura se hizo eco de esta acepción semántica de manera mucho más consistente. Se multiplicaron las referencias a la fiesta en relación con el trabajo entendiéndola como una antítesis de la actividad laboral: "Muchos domingos, al llegar a casa de doña Hortensia, me encontraba con que no había nadie, y solía entrar en el almacén. Los empleados me conocían. Allí se trabajaba lo mismo días de labor que días de fiesta" (Baroja 2002: 90).

El racionalismo materialista del siglo XX, sobre todo a partir de los años 50, ha acentuado aún más si cabe la pérdida de religiosidad formal de la fiesta y se ha iniciado una larga batalla por la conquista del tiempo libre. Sin la presión anglosajona del trabajo y sancionado por la costumbre post-industrial, el mundo festivo se ha impuesto como necesidad vital. La España del fin de siglo ha pasado a ser una sociedad consumidora, pero sobre todo productora de ocio y tiempo libre. Como decía proféticamente aquel alcarreño de El Jarama: "Cosa frecuente es esa en los madrileños, de puro desquiciados para la fiesta... Más muertos hacen las fiestas que los días de labor” (Sánchez-Ferlosio 2006: 105).

\section{LA FIESTA PENINSULAR FRENTE A LA HOLIDAY NORTEAMERICANA: ESTUDIO DIACRÓNICO}

Etimológicamente, la palabra fiesta es un descendiente semiculto del latín tardío festa, que provenía de la forma latina festum y ésta, a su vez, derivaba del adjetivo festus-a, -um. En latín clásico, la colocación más habitual de festus ocurría junto al sustantivo dia normalmente en forma plural festa dies, con el significado de jornadas en las cuales se ofrecían sacrificios en honor a un determinado dios o se celebraban juegos y banquetes públicos. El vocablo festa se mantuvo durante las invasiones bárbaras a través del latín eclesiástico, lo que explica el mantenimiento de la "f $\mathrm{f}$ " inicial y un cierto giro semántico que tendió a enfatizar el aspecto espiritual de la palabra.

Hacia el siglo XIII el uso de la forma festa se comenzó a compaginar con su variante fiesta. Ambas coexistieron durante algo más de un siglo aunque la primera seguía contando con una mayor cantidad de colocaciones en los textos que conservamos de la época (concretamente 40 casos frente a 21 según el CORDE de la R.A.E entre 1200 y 1300). La alternancia léxica deja entrever, no obstante, una cierta variabilidad connotativa. Como señala Corominas (1991), la tradicional festa parecía referirse más al espíritu y se encontraba usualmente en contextos de lengua más cultos: "El día de la festa del arcángel precioso era el mar más quedo, yazié más espacioso” (Berceo 2002: 111) mientras que la fiesta servía para describir situaciones más paganizantes: "Quando Salomón fizo el templo consagrar, vinieron 
grandes gentes la fiesta celebrar" (Ruíz 1990: 60). A partir del siglo XIV la forma festa dejó definitivamente paso a fiesta y su consolidación supuso no sólo abarcar el campo semántico de la festa, sino también reafirmar su significado como "alegría, regocijo y diversión" que se incorporará en lo sucesivo como primera acepción en glosarios y diccionarios. Covarrubias (1995: 409) en la primera definición de fiesta se hace eco de este sentimiento: "Los gentiles tenían sus días de fiesta, en los cuales ofrecían sacrificios a sus vanos dioses... y comúnmente decimos, cuando hay regocijos, que se hacen fiestas", pero también se refiere a la fiesta religiosa: "La Iglesia Católica llama fiesta la celebridad de las pascuas y los domingos y días de los santos”. El primer diccionario de la Academia de 1732 recoge una ampliación del espectro de la palabra fiesta, que pasa a cinco entradas, indicio de su creciente relevancia en la lengua y en la sociedad. Continúa apareciendo como primer significado: "Alegría, regocijo o diversión..." pero la novedad es la incorporación de la fiesta pública y popular: "Se llama también el regocijo público que se hace con el concurso del Pueblo" y la aparición del sustantivo en forma plural con dos significados: "La Pascua y sus vacaciones...los agasajos que se hacen para ganar la voluntad de alguno". Además de las definiciones anteriores, el diccionario de 1732 nos proporciona las siguientes colocaciones y locuciones que remarcan la existencia de un poder real y un poder religioso en la celebración de la fiesta:

- Fiesta doble: la que la Iglesia celebra con rito doble.

- Fiestas inmobles: las que la Iglesia celebra en ciertos y determinados días.

- Fiestas movibles: las que celebra la Iglesia en diferentes días del año.

- Fiestas reales: los festejos que se hacen en obsequio de alguna persona real, o en su presencia.

- Fiesta de guardar: el primer Mandamiento de la Iglesia.

- Estar de fiesta: estar alegre, gustoso y de chiste.

- No estar para fiestas: estar desazonado y enfadado, o no gustar de lo que se propone.

- Echar las fiestas: publicar el párroco en la misa del domingo.

Las siguientes versiones de los diccionarios de la Academia durante los siglos XIX y XX mantienen en esencia las acepciones anteriores con ligeras ampliaciones:

- Fiesta de armas: antiguamente, combate público de unos caballeros con otros para mostrar su valor (1803).

- Guardar las fiestas / santificar las fiestas: ocuparlas en cosas de Dios (1817).

- Hacer fiestas: dejar la labor o el trabajo algún día como si fuera de fiesta (1817).

- Holgar la fiesta: faltar a misa (1817).

- Quien te hace fiestas o te quiere engañar o te ha de menester: cuidado con los aduladores (1817).

- Coronar la fiesta: completarla con un hecho notable. Suele usarse irónicamente (1852).

- Aguar la fiesta: turbar cualquiera especie de regocijo (1884).

- Tengamos la fiesta en paz: expresión que se emplea para pedir a una persona que no dé motivo de disturbio (1884).

La palabra holiday deriva de la combinación de las formas del inglés antiguo hãlig y dæeg. La partícula hãlig provenía del xailaz proto-germánico, equivalente al sanctus 
en las lenguas románicas, cuyo primordial significado parecía ser "buen augurio" o “inviolabilidad" mientras que dæeg descendía directamente del dia latino. Debido a la inconsistencia de los copistas medievales en la separación de palabras resulta difícil concluir si ambos elementos aparecían juntos hãligdæeg o separados hãlig y dæeg. Hacia finales del siglo XIV en la forma combinada hãligdreg la ã no se redondeó en ô sino que se acortó en $a$ lo que dio lugar a haliday/halidai : "Iesus went himfor to plai With childir on a halidai" (Kuhn 1966: 450). Como apunta Room (1986: 84), el significado de halidai se centraba más en la noción de día de descanso y diversión, dejando la fiesta religiosa para la forma no combinada. En la forma no combinada la a larga se convirtió en $o$ corta dando lugar a holyday/holiday cuyo uso aparecía fundamentalmente ligado a contextos religiosos: "Hyt fille upon an holiday_at Sabot hight in Iewis lay" (Bogholm 2008: 172/11929), pero su empuje consiguió paulatinamente engullir a haliday. A partir del 1500 holy day y holiday coexistieron en el vocabulario popular con significados diferentes: el primero de carácter religioso y el segundo de carácter secular.

El primer vocabulario de la lengua publicado en los Estados Unidos, A School Dictionary en 1798 no recoge la palabra holy-day ni por supuesto holiday. En el glosario de Elliot de 1800, sin embargo, ya aparece el término holy-day, aunque con una única definición: “anniversary, feast, day of joy". El Noah Webster's American dictionary of the English language (1828) recoge las siguientes acepciones de holiday:

- "A day set apart for commemorating some important event in history".

- "A festival intended to celebrate some event deemed auspicious to the welfare of a nation”.

- "An anniversary festival, devoted to religious solemnities; as Christmas holydays".

- "A day of joy and gayety".

- "A day of exemption from labor; a day of amusement".

Si establecemos una comparación con las correspondientes acepciones que presentaban los diccionarios contemporáneos de la Real Academia, lo primero que salta a la vista es la mayor variedad semántica de la fiesta española ya en el siglo XIX, expresada en un mayor número de entradas y frases hechas. Si bien en ambas lenguas se menciona el carácter religioso de la fiesta, así como su connotación de alegría y diversión, los diccionarios norteamericanos hablan de ausencia de trabajo y de contribución al bienestar del país, mientras que las definiciones españolas se refieren a actividades públicas y populares. Tomando otros diccionarios norteamericanos posteriores, observaremos que las diferencias de significado que ofrecen son mínimas, fruto de las escasas variaciones semánticas que sufrirá la palabra holiday a lo largo de los primeros 60 años del siglo XIX. Los cambios semánticos se precipitarán en las últimas décadas del siglo XIX coincidiendo con el fuerte proceso de secularización que experimentó la sociedad norteamericana. Los aspectos más profanos de la fiesta se superpondrán al sentimiento religioso, lo que supondrá en los diccionarios el arrinconamiento de la entrada "holy day" en favor de holiday. En The Clarendon dictionary (1898) holiday es la única entrada del vocablo y se define en términos de: "festival day, a day of amusement" mientras que la edición de 1916 muestra muy claramente el cambio de significado que ha experimentado la palabra: "Originally, a religious anniversary; now a day set apart for exemption from labor or for a formal or informal celebration”. 


\section{LA FIESTA EN LOS DICCIONARIOS NORTEAMERICANOS Y ESPAÑOLES CONTEMPORÁNEOS}

Comparando las definiciones de la fiesta en los diccionarios de inglés americano y español peninsular se observarán interesantes diferencias de significado. Para el español peninsular se han utilizado los diccionarios Diccionario de Uso del Español (María Moliner, M.M), el Diccionario de Español (Manuel Seco, M.S), el Diccionario Vox (VOX) y el Diccionario de la Real Academia Española (R.A.E), en ese orden. Las primeras definiciones en castellano son:

- "Reunión de gente en algún sitio para divertirse o pasar agradablemente el tiempo unos con otros” (M.M).

- "Día en que, por ser domingo o por celebrarse una solemnidad religiosa o civil, no se trabaja y están cerradas las oficinas y ciertos establecimientos públicos"

(M.S).

- "Día del año eclesiástico de mayor solemnidad y en que los fieles tienen obligación de oír misa, y de abstenerse de trabajos serviles" (VOX).

- "Alegría, regocijo o diversión” (R.A.E).

En estas primeras acepciones ya se apuntan las características más relevantes de la fiesta española. Se observa una permanente mezcla de elementos religiosos y profanos (espiritualidad y diversión), pero especialmente se justifica el concepto de fiesta como antítesis del trabajo. Algunos diccionarios parecen encuadrar la fiesta en un espacio temporal, mientras otros se refieren a la felicidad de un grupo humano en general. La segunda acepción, por el contrario, presenta un mayor énfasis en la temporalidad de lo religioso y sus diferentes manifestaciones, aunque María Moliner continúe hablando de fiesta mundana y la R.A.E. siga refiriéndose a la ligereza de la broma:

- "Conjunto de actos extraordinarios...con que se celebra en una casa un acontecimiento familiar” (M.M).

- "Día en que la Iglesia conmemora un misterio, u honra de manera especial a Dios, la Virgen o algún santo” (M.S).

- "Día en que la Iglesia celebra la memoria de un santo"(VOX).

- "Chanza, broma" (R.A.E).

En la tercera entrada aparecen los conceptos de fiesta nacional y de celebración pública o popular excepto en el de la Real Academia:

- "Conjunto de actos y diversiones que se organizan en algún sitio para regocijo público, con motivo de algún acontecimiento o en fechas señaladas del año" (M.M).

- "Día laborable destinado a exaltar algo" (M.S).

- "Día en que se celebra alguna solemnidad nacional o civil" (VOX).

- "Día que la Iglesia celebra con mayor solemnidad que otros, mandando oír misa en él y emplearlo en obras santas" (R.A.E).

Las últimas definiciones explican situaciones definitivamente modernas como las vacaciones escolares o la asociación de la fiesta con actos sexuales: 
- "Caricia o demostración de cariño" (M.M).

- "Acto sexual" (M.S).

- "Reunión de gente para celebrar algún suceso, o simplemente para distraerse/

Vacaciones que se guardan por Navidad, Pascuas, etc."(VOX).

- "Vacaciones que se guardan en la fiesta de Pascua y otras solemnes" (R.A.E).

Para analizar las definiciones de la palabra holiday en la literatura léxica norteamericana, se han utilizado el Oxford English Dictionary (O.E.D) en su versión estadounidense, el Webster's College Dictionary (W.C.D), el The American Heritage Dictionary (A.H.D) y el Encarta World English Dictionary (E.W.E.D). Veamos la primera definición que nos ofrecen los diccionarios estadounidenses:

- "A consecrated day, a religious festival" (O.E.D).

- "A day fixed by law or custom on which ordinary business is suspended in commemoration of some event” (W.C.D).

- "A day free from work that one may spend at leisure, especially a day on which custom or the law dictates a halting of general business activity to celebrate a particular event" (A.H.D).

- "A day taken off or set aside for leisure and enjoyment, when somebody is exempt from work or normal activity” (E.W.E.D).

Este primer significado se centra particularmente en el hecho que se suspendan las actividades laborales para conmemorar algún día señalado. Si comparamos esta primera acepción en los diccionarios norteamericanos con su correspondencia española, observaremos que no se hace el mismo hincapié ni en la religiosidad de la jornada ni en la alegría que expresaban los diccionarios españoles. Se ve más como una obligación impuesta por la tradición que como una manifestación común nacida de la voluntad popular. La segunda definición continúa afirmando la holiday como un tiempo de descanso laboral y sólo tangencialmente aparece mencionada su conexión espiritual (los glosarios españoles centraban su segunda definición en la fiesta religiosa). Mención aparte merecen las dos acepciones que asocian la holiday con vacaciones, pero especifican que se trata de un uso particular del inglés británico:

- "A day on which ordinary occupations are suspended" (O.E.D).

- "Any day of exemption from work/ Vacation in the U.K" (W.C.D).

- "A religious feast day; a holy day"(A.H.D).

- "In U.K. vacation" (E.W.E.D).

Finalmente, la tercera definición sigue insistiendo en los mismos conceptos anteriores: vacaciones, recreo, cese de trabajo:

- "Cessation from work; festivity; recreation" (O.E.D).

- "A period of exemption from burden" (W.C.D).

- "Chiefly British, a vacation" (A.H.D).

- "A day set aside by law or statute as exempt from regular labor or business activities" (E.W.E.D). 
En definitiva, la holiday norteamericana en inglés norteamericano se define primordialmente como un día de no trabajo y en menor medida se cita la celebración religiosa, el momento de alegría o una cierta euforia, mientras que en español la fiesta parece vascular entre la alegría mundana, la celebración popular y la solemnidad del espíritu religioso, pero siempre con la antítesis del trabajo como trasfondo.

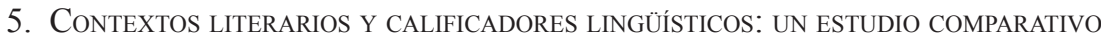

La penúltima sección de este artículo pretende encontrar pruebas semánticas en la propia estructura de las dos lenguas que confirmen la diferenciación conceptual entre holiday y fiesta. En primer lugar, se llevará a cabo una comparación estadística de los diferentes significados que expresan las palabras clave en las muestras literarias recogidas. Para examinar el uso diacrónico que las palabras clave han tenido en la lengua literaria a lo largo de la historia se ha recurrido a la ayuda de los córpora lingüísticos. En español, se ha utilizado el corpus diacrónico que la Real Academia ofrece por internet (C.O.R.D.E) mientras que en inglés norteamericano, a falta de un corpus general, se han utilizado las compilaciones textuales de William A. Williams, Jr. bajo el título de Concordances of Great Books de libre acceso en la dirección www.concordance.com. Las muestras literarias estadounidenses son un total de 170, provenientes de 17 autores norteamericanos que abarcan los siglos XVIII, XIX y XX mientras que los ejemplos recogidos en español alcanzan los 237 casos de 57 autores repartidos a lo largo de siete siglos, del XIII al XX. ${ }^{8}$

En segundo lugar, se estudiará el cambio semántico que han experimentado los vocablos a través de la historia, cotejando sus divergencias y semejanzas para, finalmente, estudiar en profundidad tanto los calificativos y modificadores que anteceden o preceden a las palabras clave como sus colocaciones predicativas. Es decir, atendiendo a criterios de variación sincrónica, de evolución diacrónica de los vocablos y de la frecuencia de aparición de las colocaciones nominales y verbales, se comprobará si lingüísticamente se siguen manteniendo las distinciones antropológicas, históricoliterarias y sociolingüísticas que hasta ahora hemos apuntado en secciones anteriores.

\subsection{Evolución diacrónica del significado de holiday y fiesta y campos semánticos ${ }^{9}$}

Mediante el estudio de los cambios semánticos en los que incurre un lexema, se pueden constatar las transformaciones cognitivas de los conceptos de holiday y fiesta.

8 Las muestras estadounidenses son por razones obvias más recientes: las primeras corresponden a los escritos de Franklin durante el siglo XVIII y las últimas llegan hasta el teatro de postguerra del siglo XX. Por lo que respecta a la selección de autores y obras se ha organizado en torno a varios criterios. En primer lugar se buscaba una cierta representatividad del autor en su cultura por lo que se han escogido escritores y escritoras que fueran reflejo de su sociedad y que a la vez la hubiesen influenciado de algún modo. En segundo lugar se ha intentado recoger textos pertenecientes a diferentes géneros (narrativa, lírica, cuento corto, etc.) para recoger en lo posible todos los contextos literarios donde pudiese aparecer la palabra clave.

9 a. Concordancias extraídas de los siguientes autores estadounidenses: Franklin, Fennimore, Poe, Cooper, Dickinson, Emerson, Douglas, Thoreau, Hawthorne, Melville, Alcott, Bowles, Twain, Alger, Whitman, James, Sinclair, Wharton, Grey. b. Concordancias extraídas de los siguientes autores españoles: Anónimo (siglos XIII-XIV), Juan Manuel, Arcipreste de Hita, Díaz de Toledo, Juan de Mena, Guevara, Fernando de Rojas, Rodríguez Montalvo, Valdés, Luján, Mateo Alemán, Cervantes, Zayas y Sotomayor, Juan de Zabaleta, Isla, Torres Villaroel, Montegón, García Malo, Moratín, Fernán Caballero, Valera, Pereda, Pérez Galdós, Clarín, Ganivet, Pardo Bazán, Blasco Ibáñez, Baroja, Unamuno, Azorín, Valle Inclán, Gironella, Poncela, Sender, Fernández Flórez, Sánchez Ferlosio, Delibes, Cela, Martín Santos, Aub, Marsé, García Hortelano, Torrente Ballester, Gironella, Goytisolo. 
El aspecto celebrativo de la fiesta peninsular ha ido ganando paulatinamente terreno por lo que respecta a su preeminencia semántica. A excepción de las colocaciones del siglo XIII, donde el modo religioso dominaba en los textos, la frecuencia de la fiesta como celebración pagana ha sumado más apariciones que el resto de los significados juntos y su influencia ha ido creciendo secularmente. Por lo que respecta al sentido religioso del término, ha pasado de una posición de fuerza durante la Edad Media y los siglos XV-XVI a un marcado retroceso a partir del siglo XVII, aunque este descenso no consigue arrebatar su lugar relevante dentro del campo semántico. El descanso laboral entró tímidamente a formar parte de la fiesta en las postrimerías del Siglo de las Luces y ha ido consolidándose como segundo valor de la palabra debido a los cambios industriales acaecidos a finales del XIX y durante el siglo XX. Los toros, aunque ocupando siempre un espacio marginal, alcanzaron su apogeo en el Siglo de Oro, pero su porcentaje fue descendiendo hasta convertirse en casi anecdótico en los textos modernos del siglo XX. Por último, cabría reseñar que la escuela y la fiesta no parecen relacionarse mucho en las muestras de lengua analizadas ya que casi no se atisba su existencia en la estadística.

Gráfico 1. Evolución del campo semántico de la palabra fiesta

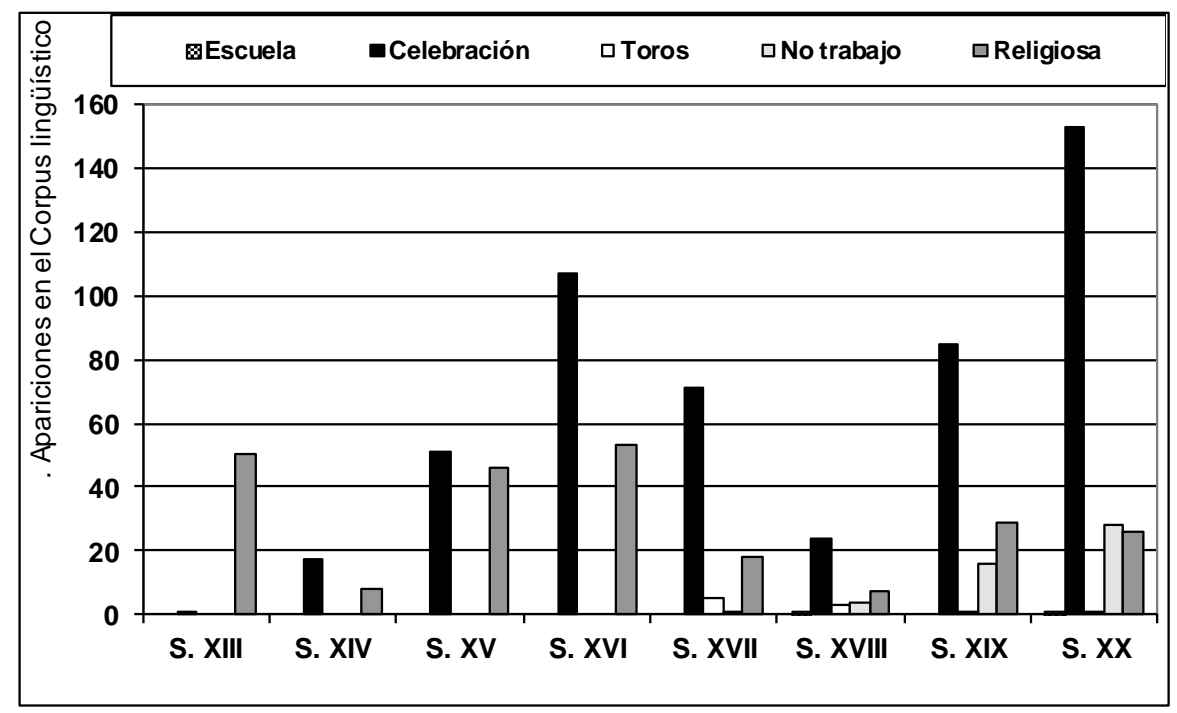

Por lo que respecta a las variaciones históricas de la palabra holiday, el descanso laboral es sin lugar a dudas la primera asociación que se realiza con el vocablo holiday durante los siglos $X I X$ y $X X$. Su frecuencia era notablemente mayor que los demás significados durante la revolución capitalista y se ha ido ampliando en la actual sociedad del ocio. El aspecto celebrativo se ha ido disociando poco a poco de la palabra en los últimos años poniendo de manifiesto una curiosa reticencia a la inclinación festiva. Por su parte, la connotación religiosa y la simbiosis escuela/ holiday también han ido cediendo terreno, aunque menos dramáticamente que en el caso del español, desde la abundancia que gozaban ambos conceptos en el siglo XIX. Finalmente, la equivalencia muerte/guerra con la fiesta que se daban en dicho siglo desaparece demostrando su estrecha relación con el empuje industrial. 
Gráfico 2. Evolución del campo semántico de la palabra holiday

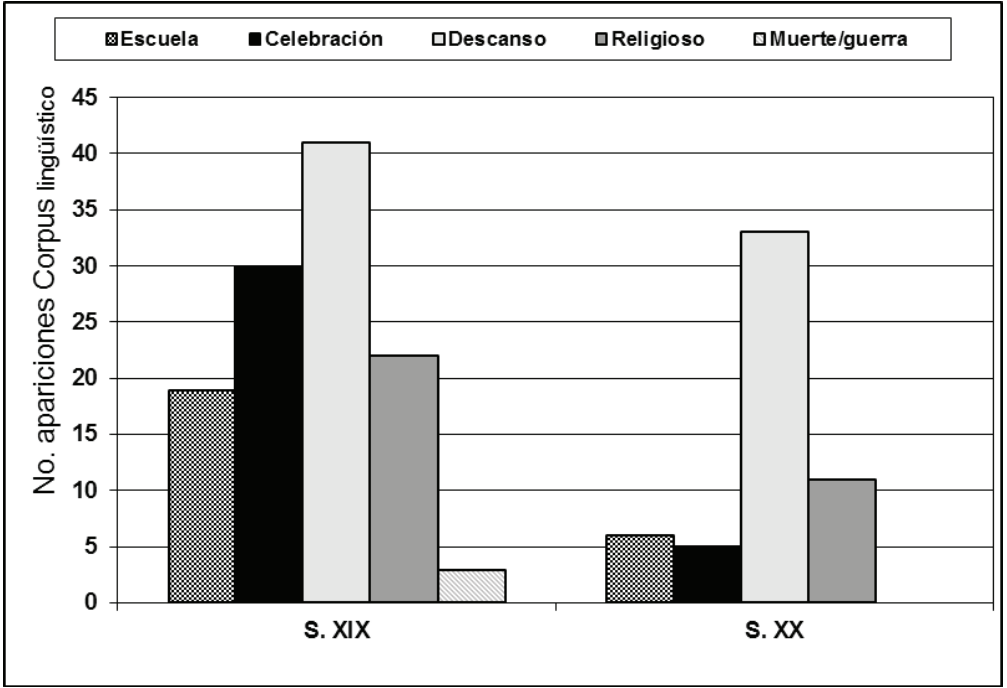

Al comparar los campos semánticos, destaca la abrumante identificación de la fiesta española con la celebración lúdica (64\%) frente a un ostensiblemente menos expansivo $21 \%$ de la holiday norteamericana. Por el contrario, en inglés, el descanso, el tiempo libre de trabajo, es la asociación más frecuente (43\%), muy superior al español que apenas consigue llegar a un 6\%. La religiosidad parece ocupar un lugar importante en ambos contextos, pero es más relevante en las muestras peninsulares (29\%) que en las estadounidenses (19\%). Finalmente, cabría destacar la importante presencia de las vacaciones escolares como sinónimo de holiday (15\%) y su aparente inexistencia en la fiesta, al menos en los contextos estudiados. Antes de concluir este subapartado no podemos dejar de mencionar dos grupos, como son el de los toros y la muerte. Ambos reflejan el riesgo en la vida pero, mientras que el español incorpora la alegría del espectáculo, el inglés se acerca al dramatismo de la guerra.

Gráfico 3. Distribución semántica del sustantivo fiesta

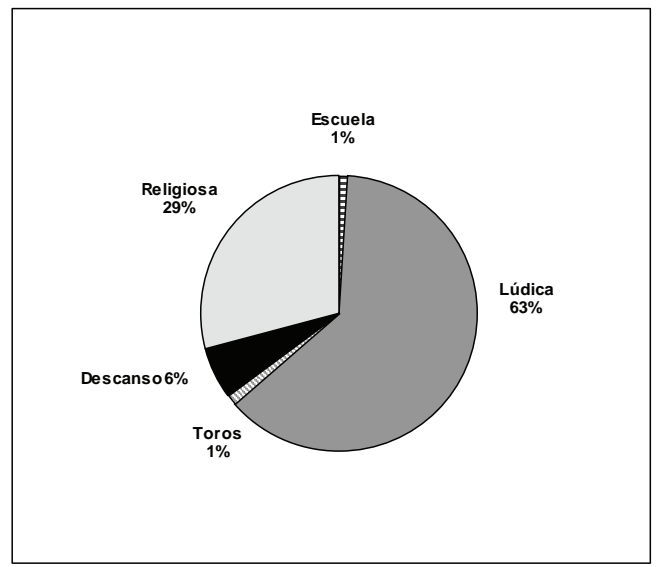


Gráfico 4. Distribución semántica del sustantivo holiday

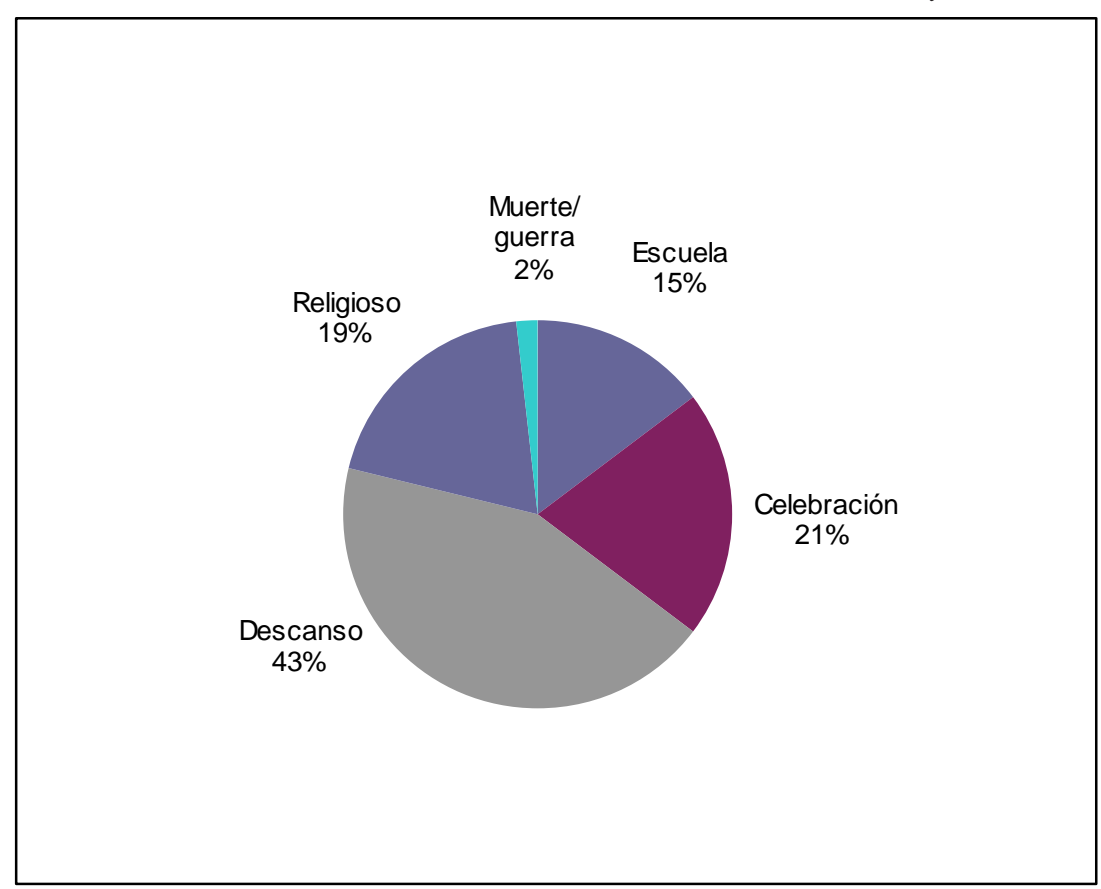

\subsection{Las colocaciones nominales y predicativas de la fiesta}

Los adjetivos y sustantivos calificadores que acompañan a la holiday y la fiesta también proporcionan pistas lingüísticas sobre la palabra a la que complementan. He aquí una lista de las colocaciones en orden decreciente:

Tabla 1. Frecuencia de calificativos y complementos nominales de fiesta/holiday

\begin{tabular}{|c|c|c|}
\hline Español & Presencia & \% \\
\hline gran/grande & 33 & 24.6 \\
\hline solemne & 11 & 8.2 \\
\hline primera & 5 & 3.7 \\
\hline santa & 4 & 3.0 \\
\hline religiosa & 4 & 3.0 \\
\hline pasada & 3 & 2.2 \\
\hline mayor & 3 & 2.2 \\
\hline doble & 3 & 2.2 \\
\hline verdadera & 3 & 2.2 \\
\hline dita/dicha & 2 & 1.5 \\
\hline celestial & 2 & 1.5 \\
\hline
\end{tabular}

\begin{tabular}{|c|c|c|}
\hline alegre & 2 & 1.5 \\
\hline divina & 2 & 1.5 \\
\hline bulliciosa & 2 & 1.5 \\
\hline mucha & 2 & 1.5 \\
\hline lucida & 2 & 1.5 \\
\hline ninguna & 2 & 1.5 \\
\hline cortesana & 2 & 1.5 \\
\hline mundana & 2 & 1.5 \\
\hline dulce & 2 & 1.5 \\
\hline mala & 1 & 0.7 \\
\hline otras & 1 & 0.7 \\
\hline alta & 1 & 0.7 \\
\hline
\end{tabular}




\begin{tabular}{|c|c|c|}
\hline buena & 1 & 0.7 \\
\hline señalada & 1 & 0.7 \\
\hline bienaventurada & 1 & 0.7 \\
\hline particular & 1 & 0.7 \\
\hline general & 1 & 0.7 \\
\hline regocijada & 1 & 0.7 \\
\hline real & 1 & 0.7 \\
\hline inopinada & 1 & 0.7 \\
\hline ordenada & 1 & 0.7 \\
\hline divulgada & 1 & 0.7 \\
\hline ocasionada & 1 & 0.7 \\
\hline sutil & 1 & 0.7 \\
\hline común & 1 & 0.7 \\
\hline secular & 1 & 0.7 \\
\hline nocturna & 1 & 0.7 \\
\hline marítima & 1 & 0.7 \\
\hline aristocrática & 1 & 0.7 \\
\hline extraordinaria & 1 & 0.7 \\
\hline peculiar & 1 & 0.7 \\
\hline naval & 1 & 0.7 \\
\hline simpática & 1 & 0.7 \\
\hline & & \\
\hline
\end{tabular}

\begin{tabular}{|c|c|c|}
\hline famosa & 1 & 0.7 \\
\hline fija & 1 & 0.7 \\
\hline estúpida & 1 & 0.7 \\
\hline sangrienta & 1 & 0.7 \\
\hline avinatada & 1 & 0.7 \\
\hline patriótica & 1 & 0.7 \\
\hline mortal & 1 & 0.7 \\
\hline estruendosa & 1 & 0.7 \\
\hline celebre & 1 & 0.7 \\
\hline nueva & 1 & 0.7 \\
\hline hermosa & 1 & 0.7 \\
\hline nacional & 1 & 0.7 \\
\hline juvenil & 1 & 0.7 \\
\hline íntima & 1 & 0.7 \\
\hline callejera & 1 & 0.7 \\
\hline pequeña & 1 & 0.7 \\
\hline personal & 1 & 0.7 \\
\hline completa & 1 & 0.7 \\
\hline consumida & 1 & 0.7 \\
\hline fazedera & 1 & 0.7 \\
\hline TOTAL & 135 & 100 \\
\hline & & \\
\hline
\end{tabular}

\begin{tabular}{|c|c|c|}
\hline Inglés & Presencia & $\mathbf{\%}$ \\
\hline national & 3 & 4.8 \\
\hline Christmas & 3 & 4.8 \\
\hline Easter & 3 & 4.8 \\
\hline summer & 3 & 4.8 \\
\hline Saturday & 3 & 4.8 \\
\hline suits & 2 & 3.2 \\
\hline public & 2 & 3.2 \\
\hline job & 2 & 3.2 \\
\hline morning & 2 & 3.2 \\
\hline rush & 2 & 3.2 \\
\hline bold & 1 & 1.6 \\
\hline gay & 1 & 1.6 \\
\hline madder & 1 & 1.6 \\
\hline
\end{tabular}

\begin{tabular}{|c|l|l|}
\hline quainter & 1 & 1.6 \\
\hline noble & 1 & 1.6 \\
\hline system & 1 & 1.6 \\
\hline fun & 1 & 1.6 \\
\hline time & 1 & 1.6 \\
\hline single & 1 & 1.6 \\
\hline attire & 1 & 1.6 \\
\hline weather & 1 & 1.6 \\
\hline apparel & 1 & 1.6 \\
\hline garment & 1 & 1.6 \\
\hline some & 1 & 1.6 \\
\hline frolic & 1 & 1.6 \\
\hline Roman & 1 & 1.6 \\
\hline excursion & 1 & 1.6 \\
\hline
\end{tabular}




\begin{tabular}{|c|c|c|}
\hline spectacle & 1 & 1.6 \\
\hline appearance & 1 & 1.6 \\
\hline royal & 1 & 1.6 \\
\hline happy & 1 & 1.6 \\
\hline Bermuda & 1 & 1.6 \\
\hline permanent & 1 & 1.6 \\
\hline welcome & 1 & 1.6 \\
\hline trip & 1 & 1.6 \\
\hline imminent & 1 & 1.6 \\
\hline brief/frugal & 1 & 1.6 \\
\hline
\end{tabular}

\begin{tabular}{|c|c|c|}
\hline vein & 1 & 1.6 \\
\hline child & 1 & 1.6 \\
\hline swiss & 1 & 1.6 \\
\hline soldiers & 1 & 1.6 \\
\hline vision & 1 & 1.6 \\
\hline faces & 1 & 1.6 \\
\hline short & 1 & 1.6 \\
\hline spiritual & 1 & 1.6 \\
\hline many & 1 & 1.6 \\
\hline Catholic & 1 & 1.6 \\
\hline TOTAL & 62 & 100 \\
\hline
\end{tabular}

A simple vista destaca el hecho que el espectro es claramente más amplio en español (65 casos frente a 47, aunque hay que tener en cuenta su superioridad en número de contextos) lo que prueba una presencia más extensa en el vocabulario de la fiesta española que su correspondiente norteamericana. En el siguiente gráfico se muestra la distribución porcentual de las colocaciones en función de los campos semánticos a los que pertenecen:

Gráfico 5. Colocaciones nominales de holiday

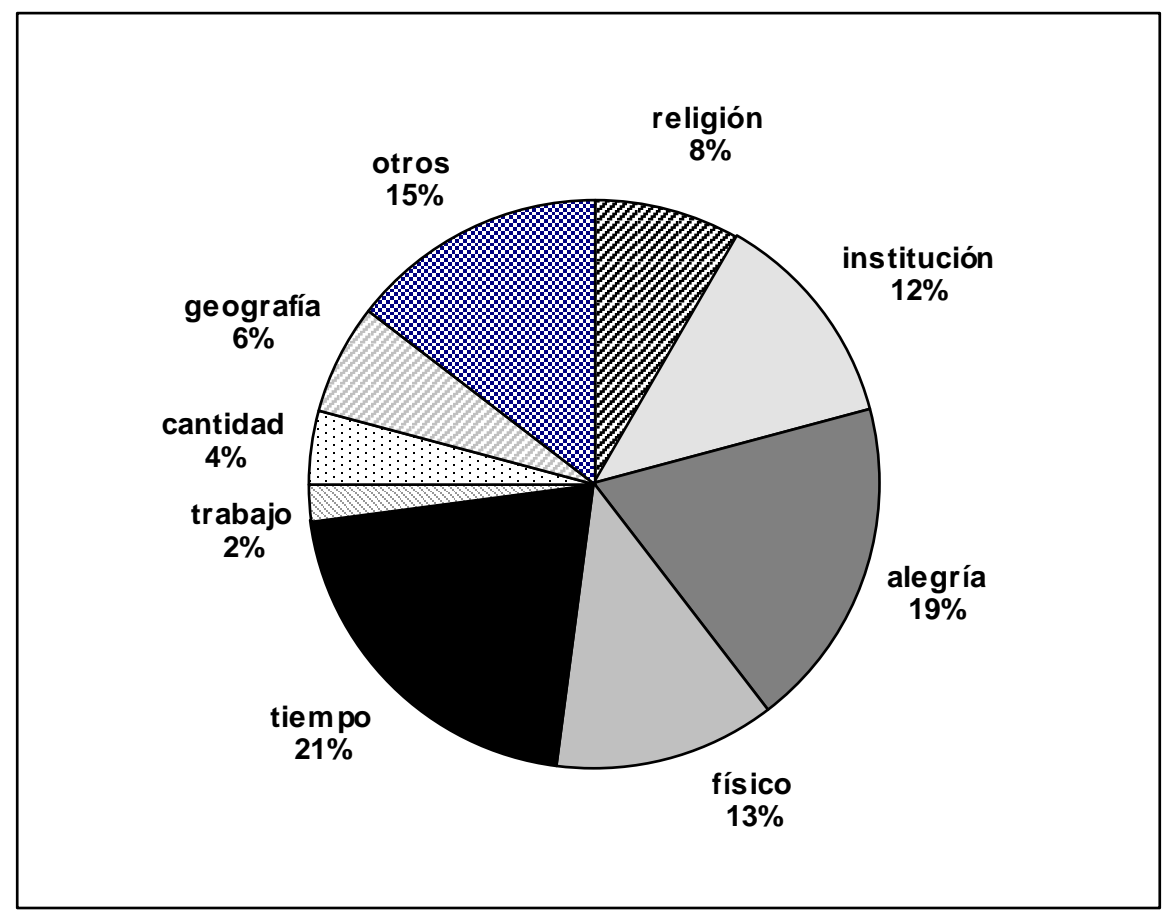


Gráfico 6. Colocaciones nominales de fiesta

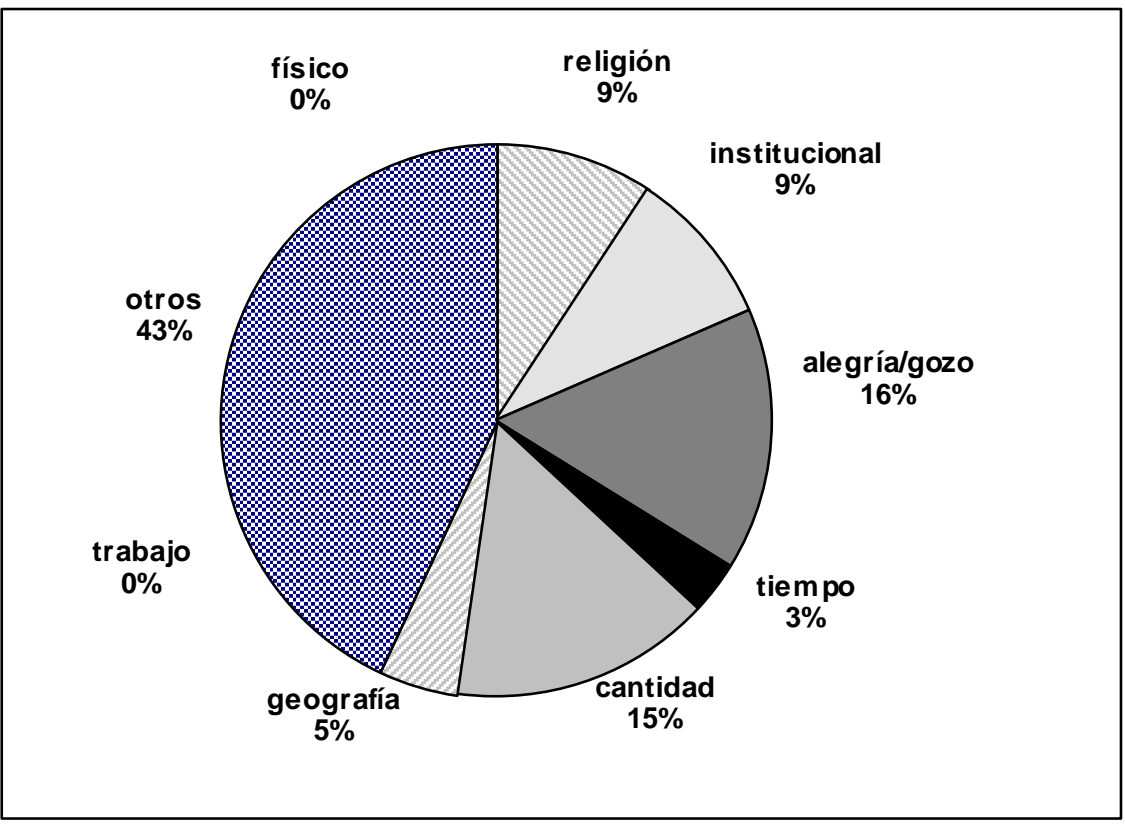

Las diferencias en el significado de las palabras que califican a holiday y fiesta no son demasiado espectaculares. Las categorías comprendidas en el campo religioso, de diversión, geográfico e institucional se asemejan extraordinariamente en sus porcentajes. Las referencias temporales son más comunes en las colocaciones norteamericanas, mientras que las cuantitativas gozan de mayor popularidad en los ejemplos sacados de la literatura española. Destacar asimismo que muchos de los calificadores del español han sido agrupados en "otros" porque ocupan demasiados ámbitos y su gran dispersión resulta inmanejable en términos comparativos. De todos modos, el mayor alcance de los campos semánticos de las colocaciones sigue apuntando a una mayor presencia en la lengua.

Para recopilar más información connotativa sobre los contextos de los vocablos se pueden analizar las cinco colocaciones más habituales de estos vocablos:

Tabla 2. Colocaciones adjetivas de fiesta/holiday

\begin{tabular}{|c|c|c|}
\hline $\begin{array}{c}\text { Colocaciones } \\
\text { inglés } \\
\text { adjetivos }\end{array}$ & $\begin{array}{c}\text { Total } \\
\text { en el } \\
\text { corpus }\end{array}$ & $\%$ \\
\hline national & 3 & $4.8 \%$ \\
\hline Christmas & 3 & $4.8 \%$ \\
\hline Easter & 3 & $4.8 \%$ \\
\hline summer & 3 & $4.8 \%$ \\
\hline Saturday & 3 & $4.8 \%$ \\
\hline
\end{tabular}

\begin{tabular}{|c|c|c|}
\hline $\begin{array}{c}\text { Colocaciones } \\
\text { español } \\
\text { adjetivos }\end{array}$ & $\begin{array}{c}\text { Total } \\
\text { en el } \\
\text { corpus }\end{array}$ & \% \\
\hline gran/grande & 33 & $24.6 \%$ \\
\hline solemne & 11 & $8.2 \%$ \\
\hline primera & 5 & $3.7 \%$ \\
\hline santa & 4 & $3.0 \%$ \\
\hline religiosa & 4 & $3.0 \%$ \\
\hline
\end{tabular}


Estadísticamente, en inglés estas colocaciones son menos representativas, ya que sólo suponen un $24 \%$ del total, mientras que en español llegan casi a la mitad (42.5\%). En cuanto a su pertenencia a grupos de significado, en inglés dos se refieren a motivos religiosos (Christmas, Easter), otras dos hablan de periodos de no trabajo (summer, Saturday) y la última expresa una cierta connotación institucional (national). En español, por el contrario, tres aluden al hecho espiritual (solemne, santa, religiosa), una al tiempo (primera) y la más numerosa incide en la calidad de la fiesta (gran/ grande). Es decir, las cinco colocaciones más frecuentes siguen ahondando en las diferencias culturales entre ambos vocablos: la holiday es religiosa, de descanso laboral e impuesta por la ley o costumbre, mientras que la fiesta es religiosa, variada en manifestaciones y abundante en cantidad.

Antes de concluir este apartado dedicado a las colocaciones, convendría profundizar en el análisis léxico de las formas verbales que rigen los predicados de nuestros conceptos claves:

Tabla 3. Complementos predicativos de fiesta/holiday

\begin{tabular}{|c|c|c|c|c|c|}
\hline Español & Presencia & $\%$ & enderezarse & 1 & 0.4 \\
\hline hazer/fazer & 48 & 21.3 & oir/ouvir & 1 & 0.4 \\
\hline celebrar & 28 & 12.4 & publicar & 1 & 0.4 \\
\hline $\mathrm{ir} /$ venir & 19 & 8.4 & canonizar & 1 & 0.4 \\
\hline acabar/terminar & 11 & 4.8 & acrecentar & 1 & 0.4 \\
\hline vestirse de & 11 & 4.8 & festejar & 1 & 0.4 \\
\hline aguar & 8 & 3.5 & cantar & 1 & 0.4 \\
\hline tener en paz & 8 & 3.5 & solazar & 1 & 0.4 \\
\hline passar & 5 & 2.2 & romper & 1 & 0.4 \\
\hline honrar & 4 & 1.7 & autorizar & 1 & 0.4 \\
\hline comenzar/empezar & 4 & 1.7 & dilatar & 1 & 0.4 \\
\hline gozar & 4 & 1.7 & cesar & 1 & 0.4 \\
\hline tener & 3 & 1.3 & admirar & 1 & 0.4 \\
\hline alegrar & 3 & 1.3 & correr & 1 & 0.4 \\
\hline estar de/andar de & 3 & 1.3 & seguir & 1 & 0.4 \\
\hline aproximarse & 3 & 1.3 & ensangrentar & 1 & 0.4 \\
\hline guardar & 2 & 0.8 & tomar a & 1 & 0.4 \\
\hline solepnizar & 2 & 0.9 & dejar & 1 & 0.4 \\
\hline regocijar & 2 & 0.8 & volver a & 1 & 0.4 \\
\hline ver & 2 & 0.8 & concluir & 1 & 0.4 \\
\hline repetir & 2 & 0.8 & turbar & 1 & 0.4 \\
\hline parecer & 2 & 0.8 & continuar & 1 & 0.4 \\
\hline anunciar & 2 & 0.8 & amenizar & 1 & 0.4 \\
\hline aplazar & 2 & 0.8 & aprovechar & 1 & 0.4 \\
\hline cumplir & 1 & 0.4 & organizar & 1 & 0.4 \\
\hline
\end{tabular}




\begin{tabular}{|c|c|c|}
\hline faltar a & 1 & 0.4 \\
\hline abandonar & 1 & 0.4 \\
\hline mirar & 1 & 0.4 \\
\hline durar & 1 & 0.4 \\
\hline dar & 1 & 0.4 \\
\hline salir de & 1 & 0.4 \\
\hline improvisar & 1 & 0.4 \\
\hline gustar & 1 & 0.4 \\
\hline prolongar & 1 & 0.4 \\
\hline observar & 1 & 0.4 \\
\hline dividir & 1 & 0.4 \\
\hline asistir a & 1 & 0.4 \\
\hline alcanzar & 1 & 0.4 \\
\hline sumergir & 1 & 0.4 \\
\hline evocar & 1 & 0.4 \\
\hline preparar & 1 & 0.4 \\
\hline fastidiar & 1 & 0.4 \\
\hline acordar & 1 & 0.4 \\
\hline aparejar & 1 & 0.4 \\
\hline destorvar & 1 & 0.4 \\
\hline llamar & 1 & 0.4 \\
\hline TOTAL & 225 & 100 \\
\hline
\end{tabular}

\begin{tabular}{|c|c|c|}
\hline Inglés & Presencia & $\mathbf{\%}$ \\
\hline take & 6 & 17.1 \\
\hline have & 5 & 14.3 \\
\hline give & 3 & 8.6 \\
\hline ask for & 3 & 8.6 \\
\hline spend & 2 & 5.7 \\
\hline get & 2 & 5.7 \\
\hline carry & 1 & 2.9 \\
\hline take place & 1 & 2.9 \\
\hline glaze & 1 & 2.9 \\
\hline employ & 1 & 2.9 \\
\hline reflex & 1 & 2.9 \\
\hline go on & 1 & 2.9 \\
\hline make ready & 1 & 2.9 \\
\hline enjoy & 1 & 2.9 \\
\hline make & 1 & 2.9 \\
\hline be taken & 1 & 2.9 \\
\hline steal & 1 & 2.9 \\
\hline beg & 1 & 2.9 \\
\hline be refused & 1 & 2.9 \\
\hline want & 1 & 2.9 \\
\hline TOTAL & 35 & 100 \\
\hline & & \\
\hline
\end{tabular}

Resulta evidente que la fiesta se encuentra regida por un mayor número de predicados verbales que los hallados para holiday. Como se ha mencionado anteriormente, parte de la diferencia obedece a la desproporción de muestras en favor del significado español, pero sería miope dejar de constatar un superior grado de dispersión igual al que existe en las colocaciones nominales. La palabra holiday aparece gobernada por formas verbales que expresan fundamentalmente pertenencia (25\%), creación (15\%), petición (10\%) y tradición (10\%), mientras que su correspondiente término castellano se une a verbos que comunican ideas de diversión (23\%), tiempo (14\%), percepción (13\%) y tradición (12\%). El énfasis de cada lengua parece claro y diferenciado: las colocaciones verbales de los textos literarios estadounidenses enfatizan el sentido de pertenencia a una colectividad, en este caso semántica o sociolingüística, que organiza o recibe una celebración cuya existencia viene marcada por una costumbre arraigada en la sociedad y en el pasado. La palabra fiesta, por su parte, se enmarca en un contexto de goce o alegría dentro de unas coordenadas temporales que no duda en recrearse con los sentidos al amparo de un uso preestablecido. 
Gráfico 7. Colocaciones predicativas de holiday

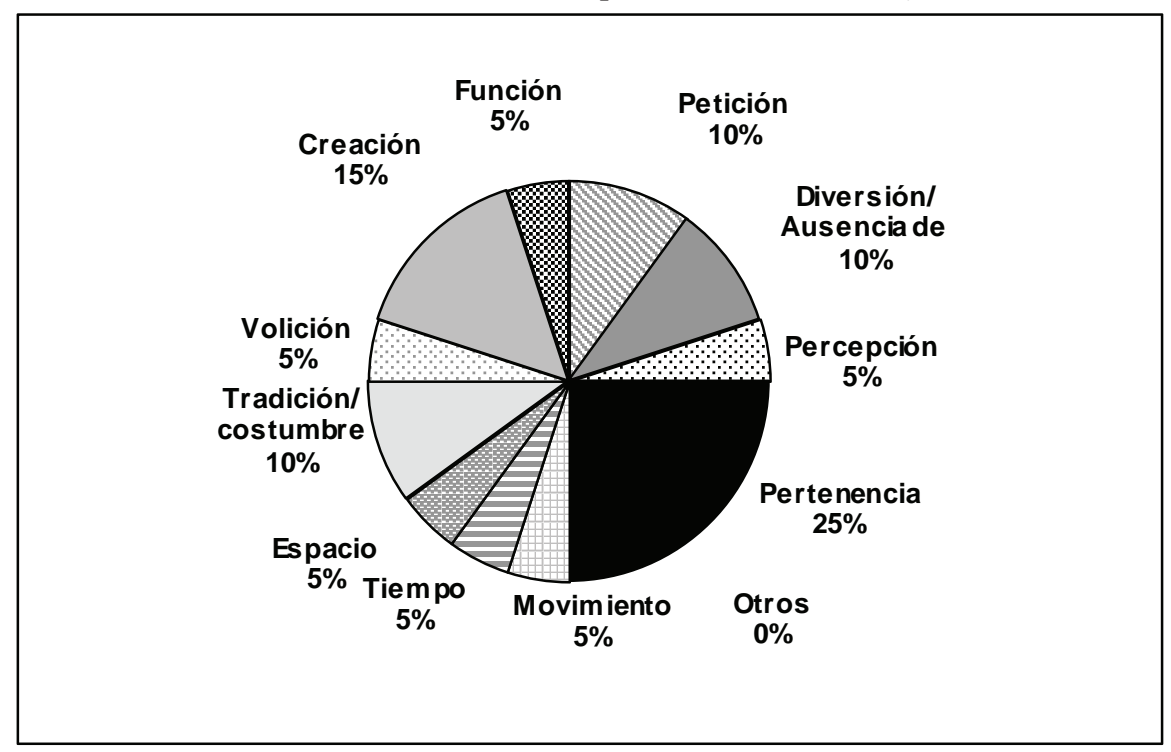

Gráfico 8. Colocaciones predicativas de fiesta

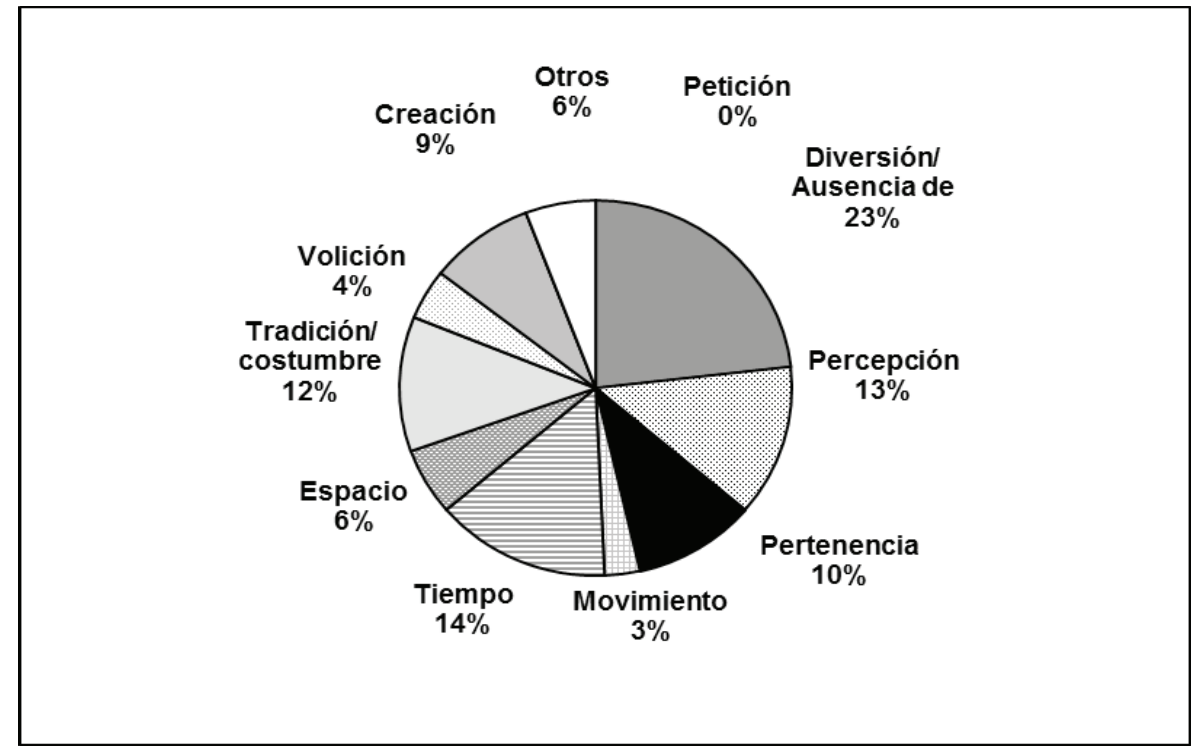

Todos estos análisis estadísticos del texto, el contexto y los lexemas parecen converger en tres hallazgos fundamentales. Por un lado, los campos semánticos ratifican una distinta caracterización de las palabras fiesta y holiday que no resulta en absoluto contradictoria con la descripción que nos ofrecía la antropología lingüística. En segundo lugar, la fiesta ha demostrado en ambos registros una importante evolución semántica 
a lo largo de los siglos, fiel reflejo de los cambios de valores que han experimentado las palabras en la sociedad. La tercera conclusión sería que las colocaciones, ya sean sustantivas o verbales, han evidenciado la mayor relevancia de la variante peninsular con respecto a la norteamericana en cuanto a frecuencia de uso y, por supuesto, han confirmado la correlación sociolingüística entre el significado de las palabras calificativas y ciertos modelos o pautas de comportamiento.

\section{CONCLUSIÓN}

A lo largo de este artículo se han ido analizando y comparando los términos fiesta y holiday con la intención de corroborar la hipótesis de partida que apuntaba a sus singularidades cognitivas, sociales y lingüísticas. Si para los antropólogos norteamericanos el mundo festivo se entiende como una peligrosa desviación del orden establecido, para los estudiosos españoles la celebración es un momento de sublimación en la existencia de cualquier sociedad. El rechazo protestante que importaron las colonias norteamericanas se contrapuso al furor festivo peninsular del Barroco español. Los caminos se alejarían aún más con la irrupción del capitalismo moderno en los Estados Unidos y con el atraso industrial que caracterizó a la España del siglo XIX. El reflejo de la fiesta palideció en una cultura norteamericana que hacía del trabajo su máxima aspiración y floreció en la península ibérica como válvula de escape de la apatía en la que se hallaba sumido el país. La revolución tecnológica del siglo XX ha acercado parcialmente los dos universos al potenciar el valor del ocio, pero el individualismo anglosajón se resiste a aceptar el valor comunitario de la fiesta.

Desde el punto de vista estrictamente lingüístico se han comparado los vocablos holiday/fiesta sincrónica y diacrónicamente. El análisis diacrónico ha contrastado las definiciones de los diccionarios a fin de determinar la evolución histórica de los significados en los glosarios norteamericanos y peninsulares. Una vez entendido el marco histórico se ha procedido a cotejar las explicaciones que nos daban los diccionarios actuales. De esta comparación se han obtenido evidencias claras de interpretaciones cognitivas diferenciadas no limitadas a cada ámbito de lengua. Ver la distinta conceptualización que efectúan los diccionarios es tan sólo uno de los posibles campos de profundización interlingüística. Finalmente, se ha llevado a cabo un barrido de las colocaciones léxicas asociadas con la fiesta. Tomando toda la lista de sustantivos, adjetivos y verbos que la calificaban se ha calculado la prominencia semántica con porcentajes al tiempo que se han establecido patrones propios de frecuencia y pertenencia de las colocaciones más habituales.

Así pues, parece evidente que traducir holiday por fiesta y viceversa sin más, conduce a un cierto grado de incomunicación cultural, ya que los dos términos plantean posiciones culturales contrapuestas. No resulta posible hablar de la existencia de axiomas antropológicos semejantes ni de un vínculo etimológico común; tampoco es plausible el argumento de la coincidencia léxica en las definiciones de los diccionarios ni tan siquiera la falacia de la convergencia de campos semánticos. Por todo ello, el estudiante español debe aceptar que la holiday norteamericana es la antítesis del trabajo y se asocia al consumismo, mientras que para el norteamericano la fiesta peninsular debe ser entendida como un crisol de experiencias comunes a medio camino entre el trasfondo espiritual y el goce mundano. 


\section{OBRAS CITADAS}

Adams, Henry. 2002. The Education of Henry Adams. Devon: Dover. Baroja, Pío. 2002. Las inquietudes de Shanti Andia. Madrid: Red.

Bell, Daniel. 1979. The cultural contradictions of Capitalism. New York: Cosimo.

Berceo, Gonzalo. 2002. Milagros de Nuestra Señora. Madrid: Crítica.

Bogholm, Niel. 2008. English speech from an historical point of view. Michigan: Nyt nordisk forlag.

Caballero, Fernán. 1998. La família de Alvareda. Madrid: Cátedra.

Chalmeta, Pedro. 1995. Cultura y culturas en la historia. Salamanca: Universidad de Salamanca.

Covarrubias, Sebastián. 1995. Tesoro de la lengua castellana o española. Madrid: Castalia.

Corominas, Joan y Pascual, José Antonio. 1991. Diccionario crítico etimológico castellano e hispánico. Madrid: Gredos.

Emerson, Ralph Waldo. 2004. Essays and poems. NY: Barnes and Nobles Classics.

Gil, Eduardo. 1998. Vida cotidiana en la España Medieval. Palencia: Santa María la Real.

Hand, William. 1902. The Clarenton Dictionary. Harvard: University Publisher.

Ibrahim, Hilmi. 1991. Leisure and society: a comparative approach. Madison: Wm. C. Brown.

Kuhn, Sherman. 1966. Middle English Dictionary. Michigan: University of Michigan Press.

Moliner, María. 1966-67 facs. 2007. Diccionario de uso del español. Madrid: Gredos. $3^{\mathrm{a}}$ ed.

Martínez-Burgos, Palma. 2004. La fiesta en el mundo hispánico. Cuenca: Universidad de Castilla la Mancha.

Real Academia Española. Diccionario de la lengua española. Fuente electrónica [en línea]. Madrid: Real Academia Española. http://corpus.rae.es/creanet.html

Room, Adrian. 1986. A dictionary of true etymologies. North Carolina: Taylor \& Francis.

Ruíz, Juan Antonio.1990. La historia de la salvación en la obra de Gonzalo de Berceo. Logroño: Instituto de Estudios Riojanos.

Sánchez-Ferlosio, Rafael. 2006. El Jarama. Madrid: Espasa.

Santino, Jack. 1994. All around the year: holidays and celebrations in American life. Urbana: University of Illinois Press.

Seco, Manuel. Et. al. 1999. Diccionario de español actual. Madrid: Aguilar. $2^{\mathrm{a}}$ ed.

Stephenson, George. 1978. The Puritan heritage. Westport: Greenwood Press.

Updyke, Winn. 1970. Principles of modern physical education, health, and recreation. Minnesota: Holt.

VV.AA. 1996. Diccionario general de la lengua española. Madrid: Vox.

VV.AA. 1994. The World Book Dictionary. Chicago: Field Enterprises Educational Corporation. $2^{\text {a }}$ ed.

VV.AA. 1990. Webster's 1828 Dictionary. ed. facs. Boston: Merriam-Webster.

VV.AA. 1989. Oxford English Dictionary. $2^{\mathrm{a}}$ ed. Oxford: Oxford University Press.

VV.AA. 1969. The American Heritage Dictionary. Boston: Houghton Mifflin.

VV.AA. 1900[1872]. Chambers English Dictionary. 1872 facs.1990. Edinburgh: Chambers Harrap Publishers. $4^{\mathrm{a}}$ ed.

Warton, Edith. 1998. New York Novels. Michigan: Modern Library. 